\title{
Similar Biological Medicinal Products Containing Recombinant Human Growth Hormone: European Regulation
}

\author{
Mira Pavlovic Elizabeth Girardin Liliana Kapetanovic Kowid Ho \\ Jean-Hugues Trouvin \\ Scientific Advice Unit, Agence Française de Sécurité Sanitaire des Produits de Santé, Saint-Denis, France
}

\section{Key Words}

Similar biological medicinal products, comparability • Somatropins

\section{Abstract}

The concept of similar biological medicinal products ('biosimilar' medicinal products) allows pharmaceutical companies to develop products based on an abridged dossier once the marketing protection of the 'reference' biological medicinal product has expired. A biosimilar medicinal product can be granted a marketing authorization provided that its similarity to a reference product is established in terms of quality, safety and efficacy (step-wise comparability exercise). A decision to launch a biosimilar medicinal product on the market is taken if it has a similar efficacy and comparable or better (less) immunogenicity than the chosen reference biological medicinal product. However, this decision is based on limited data and the comparability program may detect substantial differences in immunogenicity profiles but is likely incapable of detecting rare events. This is why clinical experience, through clinical trials and extensive pharmacovigilance programs, remains the most reliable way to assess the immunogenicity and tolerance profile of recombinant therapeutic proteins. Substitution of one biological medicinal product by a biosimilar medicinal product is not currently recommended before long-term clinical efficacy and safety have been acquired in all relevant populations. Here we
\end{abstract}

\section{KARGER}

Fax +41613061234

E-Mail karger@karger.ch

www.karger.com (c) 2007 S. Karger AG, Basel

0301-0163/08/0691-0014\$24.50/0

Accessible online at:

www.karger.com/hre review recent regulatory guidelines provided by EMEA and comment on the marketing authorizations and risk management plans of two recently approved biosimilar somatropins.

Copyright $\odot 2007$ S. Karger AG, Basel

\section{Introduction}

The concept of similar biological medicinal products ('biosimilar' medicinal products) has been recently introduced in the European legislative framework $[1,2]$. The purpose was to open a regulatory route for pharmaceutical companies willing to develop biosimilar medicinal products once the marketing protection of the 'reference' biological medicinal product (BMP) expired.

Several BMPs are or will be out of patent in the near future, which offers pharmaceutical companies the possibility to develop similar products and to obtain the same therapeutic indications as out-of-patent reference products.

Even if this strategy could be easily assimilated to the standard generic approach (which, for a chemically derived substance, requires a single demonstration of bioequivalence with the reference product), the generic approach was not considered adequate to establish the quality, safety and efficacy of biosimilar BMPs $[1,2]$. This is due to the complexity of biotechnology-derived active in- 
gredients and of their manufacturing process. In most cases, molecular complexity and inherent heterogeneity of biosimilar BMPs does not allow for their full characterization. The quality attributes of the active ingredient are highly dependent on its manufacturing process and any change in the manufacturing process may affect the quality attributes and impact on the safety or efficacy profiles of the product $[2,3]$. Therefore the European legislation has provided a specific regulatory approach (called 'biosimilar approach') for biosimilar BMPs (see table 1 for definitions).

This biosimilar approach may be applicable to any BMP. Practically, it is applied to well-characterized active ingredients, mainly recombinant proteins (e.g. insulin, somatropin, epoetin, granulocyte colony-stimulating factor). The Committee for Medicinal Products for $\mathrm{Hu}-$ man Use (CHMP) of the European Medicines Agency (EMEA) has recently published several guidelines relevant to quality, non-clinical and clinical issues to be addressed within the development of such biosimilar BMPs [2-4].

In this mini review we summarize and discuss the recommendations related to the clinical development of biosimilar somatropins [5].

\section{Regulatory Context}

Quality, safety and efficacy are key components that need to be monitored throughout the lifecycle of any medicinal product. The drug development process is well defined for 'classical', chemically derived products. In addition to pharmaceutical data for documenting the quality of the product, non-clinical data are needed before the first administration in humans. The clinical development process requires data on the proof of principle, dose finding and efficacy demonstration in the disease population. Based on quality, non-clinical and clinical data accumulated from early development through phase 3 clinical trials, the product can go through the regulatory process to seek for a marketing authorization.

As for chemically derived products, the development of a biosimilar BMP comprises the development of the manufacturing process (for both the active ingredient and the final product) and the demonstration of safety and efficacy by means of non-clinical and clinical trials. However, as the reference BMP has been authorized and used in the EU for several years, data on both the active substance and on the final product are publicly available. Therefore the development of a biosimilar BMP does not

Biosimilar Somatropins
Table 1. Definitions

Biological medicinal product (BMP): a medicinal product whose active substance is a biological substance (e.g. recombinant DNA, vaccine, blood- or plasma-derived product, monoclonal antibody). A biological substance is produced or extracted from a biological source and needs for its characterization a combination of physicochemical-biological testing as well as a fully developed production process and its control.

Biosimilar (similar) biological medicinal product (biosimilar BMP): a medicinal product developed by a new manufacturer and claimed to be similar to a known ('reference') BMP. A biosimilar BMP contains the same active substance as the reference BMP and is intended to be used for treating the same disease(s), at the same dose and using the same route of administration.

Reference biological medicinal product (reference BMP): a BMP that is authorized and marketed in the European Union.

usually require as much non-clinical and clinical data as a completely new medicinal product. In addition, the reference BMP may have more than one indication, and in certain cases it may be possible to extrapolate therapeutic similarity from the comparison between the biosimilar and the reference BMP made in one specific population/ indication to other indications of the reference BMP. This will depend on whether or not the same mechanisms of action and/or the same receptors are involved in all indications and on the clinical experience available.

The peculiarity of the biosimilar approach is the 'comparability exercise', which imposes a comparison of the biosimilar BMP to a reference BMP in terms of quality, safety and efficacy. If several reference BMP have been authorized in the EU for the same indication(s), any reference BMP may be chosen for the comparability exercise; however, the same reference BMP should be used throughout the comparability programme in order to obtain coherent data and relevant conclusions. If the CHMP approves the marketing authorization application, the indications given to a biosimilar BMP will be those of the reference BMP.

The comparability exercise follows a step-wise approach. The first step is a thorough comparison of the two products at the quality level. Any difference in the quality attributes of the biosimilar and reference BMPs may have potential implications on safety and efficacy and will influence the amount and the content of the subsequent comparative non-clinical and clinical studies ('bridging studies'). The non-clinical comparative studies usually include receptor-binding studies, cell-based 
assays, studies of pharmacodynamic activity and a restricted non-clinical toxicity programme.

The clinical comparability is also a step-wise process, which usually begins with pharmacokinetic/pharmacodynamic studies in healthy volunteers, followed by comparative clinical efficacy and safety trials. In most cases, clinical efficacy trials are performed to demonstrate therapeutic equivalence between the biosimilar and the reference BMP in the most sensitive and the most relevant patient population.

However, even if the efficacy is demonstrated in therapeutic equivalence trial(s), the safety of a biosimilar BMP may differ from that of its reference BMP due to differences in the quality attributes of both products, which may or may have not been apparent during the quality comparability exercise. Because such differences may have unforeseeable clinical consequences, the clinical safety of a new biosimilar BMP must be extensively evaluated both before and after marketing authorization.

During safety assessment, special attention is given to the immunogenicity of biosimilar BMPs, as patients may develop clinically relevant antibodies to any recombinant protein. The immunogenic potential of BMPs may differ between products and is influenced by several factors, such as the nature and structure of the active substance, impurities, excipients, manufacturing process, route of administration and target patient population. These differences may compromise the in vivo behaviour of the product, resulting in undesired host immune responses to the drug that may abrogate efficacy and may give rise to potentially fatal adverse reactions [6].

Several approaches based on algorithms integrating the epitope response to HLA polymorphism, in vitro assays (e.g. epitope mapping, cell-based assay, etc.) or animal models may be used to assess the antigenicity profile of a BMP. Although these approaches may be useful in identifying the relative antigenicity of a product during its development, the immune response in vivo cannot be predicted. Assessing the antigenicity profile in humans is also challenging since the ability to measure antibodies to recombinant proteins is often limited (e.g. human immune sera are rarely available, lack of appropriate standards, interference with the endogenous protein, limitations of analytical methods). In consequence, the comparison of results across products in the same class may lead to inaccurate interpretations $[7,8]$.

In principle, a decision to launch a biosimilar BMP on the market is taken if it has similar efficacy and at least comparable or better (less) immunogenicity than the chosen reference BMP. However, under the current guide- lines, this decision is based on rather limited data. The comparability program may detect substantial differences in immunogenicity profiles but will probably be incapable of detecting rare events. This is why clinical experience, through clinical trials and extensive pharmacovigilance programmes, remains the most reliable way to assess the immunogenicity profile of recombinant therapeutic proteins in patients. Some adverse reactions are very rare and safety assessment is necessary throughout the life cycle of the product, especially when a new biosimilar BMP is launched on the market.

\section{Somatropins}

Human growth hormone (GH) has been used for the treatment of GH deficiency in children and adults, but also in an increasing number of conditions with short stature and normal endogenous GH secretion, such as Turner syndrome, children with stunted growth due to chronic renal insufficiency, Prader-Willi syndrome and for short children born small for gestational age. In the US, but not in Europe, GH has also been used for treatment of idiopathic short stature (non-GH-deficient children). In GH-deficient patients, low (replacement) doses are generally sufficient to obtain a therapeutic effect. In contrast, in non-GH-deficient conditions such as Turner syndrome, Prader-Willi syndrome or in patients with chronic renal insufficiency, supra-physiological doses are necessary due to resistance or disturbed binding capacity of GH and insulin-like growth factor (IGF)-1.

$\mathrm{GH}$ acts by binding to $\mathrm{GH}$ receptors and produces its effects both directly and indirectly via the stimulation of IGF-1. The biodisponibility of GH is modulated not only by its binding to $\mathrm{GH}$-binding proteins but also by 6 IGFbinding proteins (IGFBPs, mostly IGFBP-3), which regulate the proportion of free IGFs [9]. More than $90 \%$ of circulating IGFs are complexed with IGFBP-3, both upregulated by GH [9]. The actions of IGFBP-3 can oppose those of IGF-1, in part by binding IGF-1 and thus limiting the availability of free IGF, but also by a direct inhibitory effect on target cells [10]. Another complexity of the system is due to the presence of various proteases that cleave the ligand from its binding protein. These include prostate-specific antigen as well as a protease for IGFBP-3. Finally, local IGF-1 bioactivity is also influenced by tissue architecture and variations in proteoglycans, perhaps as a result of their varying affinities for the different binding proteins [11].
Pavlovic/Girardin/Kapetanovic/Ho/ Trouvin 
Metabolic effects of GH are mainly due to those of IGF1 and comprise an increase in lean mass, muscular strength and cardiac output as well as a decrease in fat mass. GH also has a considerable effect on mood, which could be partly due to its action on GH receptors in the brain [12].

The safety of somatropins is generally considered satisfactory $[13,14]$. Data from old registration trials is very limited due to low numbers of patients and a lack of randomized controls. Therefore most of the currently available pharmacovigilance information has been obtained from large post-marketing studies sponsored by GH manufacturers. These studies have identified safety issues occurring during GH treatment, such as diabetes [15], slipped capital femoral epiphyses [16] and intracranial hypertension [17], but could not address issues occurring after treatment is stopped (such as neoplasms) as patients are mostly lost to follow-up once GH treatment is discontinued.

The GH/IGF-1 axis and in particular the increased IGF-1 levels might be associated with increased cancer risk [18], especially colorectal cancer. This increase in risk has been observed not only in acromegaly patients [19, 20] but also in the general population [21]. It is believed to be due to the proliferative and anti-apoptotic effects of IGF-1, which could provide an environment that favours the survival of genetically damaged cells [11]. The increased risk of malignancies in the general population [21] is a serious concern in the context of increasing use of GH in short but otherwise normal children. Therefore prospective surveillance of cancers expressing IGF-1 receptors [22-24] is recommended for all currently approved GHs [25].

\section{Biosimilar Somatropins}

In addition to general guidelines on the development of biosimilar BMPs, the EMEA has elaborated specific guidelines for each class of BMP, in particular GH [5]. As the same GH receptor is thought to be involved in all currently approved therapeutic indications of recombinant human GH (rhGH), it is considered that demonstration of similar efficacy and safety between a biosimilar and an approved somatropin in GH-deficient children may allow extrapolation to other indications of the reference somatropin. A homogenous population of treatment-naïve children with GH deficiency is recommended since this patient population is considered the most sensitive to GH and a well-known model for studying the efficacy of somatropin.

Biosimilar Somatropins
The guidelines require that clinical comparability between the biosimilar and the reference somatropin be shown in at least one adequately powered, randomized, double-blind, 2 -arm, parallel-group therapeutic equivalence trial of sufficient duration. Change in height velocity or change in height velocity standard deviation is the recommended primary efficacy endpoint. Children to be included should be pre-pubertal before and during the study to avoid interference of the pubertal growth spurt with the treatment effect. Calculation of pre-treatment growth rates should be based on observation periods of $6-18$ months before entry into the study. Due to significant variability in short-term growth rates, seasonal variability in growth and measurement errors inherent to short-term growth measurements, the recommended duration of the study is at least 6 months and, ideally, 12 months. A 12month study is also important for safety data collection, as 12-month comparative immunogenicity data are required before approval. In addition, sampling for IGF-1, IGFBP-3, fasting insulin and blood glucose is also recommended.

IGF-1 and IGFBP-3 levels should also be studied in a single-dose cross-over comparative pharmacokinetic/ pharmacodynamic study in healthy volunteers.

In addition, a detailed post-marketing surveillance plan (risk management plan) should be provided in order to cover safety issues related to the use in all target populations (indications).

In practice, the risk management plans should cover topics such as the diabetogenic potential in GH-deficient children and children born small for gestational age, the occurrence and clinical implications of anti-rhGH and anti-host-cell protein (anti-HCP) antibodies, risk of hypothyroidism, specific risk of rhGH treatment in PraderWilli syndrome patients and occurrence of malignancies due to the mitogenic and proliferative properties attributed to the activity of the GH/IGF-1 axis and increased levels of IGF-1 $[5,26,27]$.

\section{Recent Approvals}

Two biosimilar somatropins have recently been granted a marketing authorization in the European Union: Omnitrope ${ }^{\circledR}$ (January 2006) [26] and Valtropin ${ }^{\circledR}$ (February 2006) [27].

Omnitrope has also been authorized by the US Food and Drug Administration as a 'follow-on protein product' in May 2006 and was not considered as therapeutically equivalent and therefore substitutable for any of the other approved human $\mathrm{GH}$ products [28].

Horm Res 2008;69:14-21 
Table 2. Results from Omnitrope and Valtropin trials

\begin{tabular}{|c|c|c|c|}
\hline & Omnitrope & Genotropin & $95 \%$ CI \\
\hline Number of patients & 44 & 45 & \\
\hline Duration, months & 9 & 9 & \\
\hline Height baseline, $\mathrm{cm}$ & 113.3 & 109.3 & \\
\hline Height at 9 months, $\mathrm{cm}$ & 121.9 & 117.7 & $-0.59,1.06$ \\
\hline Height velocity pre-treatment, $\mathrm{cm} /$ year & 3.8 & 4.0 & \\
\hline Height velocity at 9 months, $\mathrm{cm} /$ year & 10.7 & 10.7 & $-1.35,0.92$ \\
\hline Height velocity SDS at baseline, $\mathrm{cm} /$ year & -2.4 & -2.3 & \\
\hline \multirow[t]{2}{*}{ Height velocity SDS at 9 months, $\mathrm{cm} /$ year } & 6.1 & 5.4 & $-0.81,2.13$ \\
\hline & Valtropin & Humatrope & $95 \%$ CI \\
\hline Number of patients (PP) & $98(70)$ & $49(32)$ & \\
\hline Duration, months & 12 & 12 & \\
\hline Height baseline, $\mathrm{cm}$ & 108.4 & 111.3 & \\
\hline Height at 12 months, $\mathrm{cm}$ & 120.2 & 122.5 & NS \\
\hline Height velocity pre-treatment, $\mathrm{cm} /$ year & 3.6 & 3.4 & \\
\hline Height velocity at 12 months, $\mathrm{cm} /$ year & 11.3 & 10.5 & $-0.71,0.90$ \\
\hline Height velocity SDS at baseline & -2.19 & -2.42 & \\
\hline Height velocity SDS at 12 months & 5.62 & 5.33 & NS \\
\hline
\end{tabular}

SDS = Standard deviation score $\mathrm{PP}=$ per protocol NS = not significant $\mathrm{CI}=$ confi dence interval.
The FDA term 'follow-on protein product' generally refers to protein products (produced through biotechnology or derived from natural sources) intended to be sufficiently similar to a product already approved to permit the applicant to rely for approval on certain existing scientific knowledge about the approved product. There are two possible pathways for approval of a follow-on protein product, either under approval mechanisms found in section 505 of the Food, Drug and Cosmetic (FDC) Act applicable to drugs, or under section 351 of the Public Health Service (PHS) Act applicable to biological products. Omnitrope was approved under section 505(b)(2) of the FDC Act [28], which is usually applied to generic drugs and allows for an abbreviated approval pathway. The majority of protein products are licensed as biological products under the section 351 of the PHS Act, which does not currently allow for an abbreviated approval pathway analogous to 505(b)(2) of the FDC Act. Such a pathway for the approval or licensure of follow-on protein products under the PHS Act would require new legislation. Guidance documents on the development of follow-on protein products and their approval might be issued by the FDA in the future.

The development programme of both products in Europe had been planned and performed before the EMEA guidance on biosimilar somatropins became available.
Both applications were submitted under the legal base of Similar Biological Medicinal Product (Article 10.4 of Directive 2004/27/EC). The applications contained a full quality dossier and reduced non-clinical and clinical parts, including the elements of the comparability exercise.

Both Valtropin and Omnitrope have chosen the most sensitive model (GH-deficient children) to show comparable efficacy and safety to the reference BMP and to extrapolate clinical data to all indications approved for the chosen reference BMP.

Omnitrope has been produced in an Escherichia coli host strain by recombinant DNA technology. During the clinical development programme various formulations of GH were used, with active pharmaceutical ingredient (API) as follows:

- Somatropin Sandoz powder for solution for injection (API Covance)

- Somatropin Sandoz powder for solution for injection (API Sandoz): formulation to be marketed as Omnitrope

- Somatropin Sandoz liquid (API Sandoz liquid)

The effects of Omnitrope (API Covance formulation) were compared to Genotropin in 89 pre-pubertal, GH-deficient, treatment-naive children in an open 9-month trial. At the end of treatment, increases in height were comparable in both treatments: from 113.3 to $121.9 \mathrm{~cm}$ with 
Omnitrope and from 109.3 to $117.7 \mathrm{~cm}$ with Genotropin (see table 2). During the trial, up to $60 \%$ of the enrolled patients had developed anti-GH antibodies without showing any influence on growth rate. Careful investigation revealed high concentrations of HCPs leading to development of anti-HCP antibodies, known to enhance the antibody reaction against $\mathrm{GH}$. The manufacturing process for Omnitrope was therefore modified by introducing additional purification steps during the development of the product. The concentrations of HCPs in the subsequent formulations (API Sandoz and API Sandoz liquid) were within the range known from other authorized GH-containing products. Subsequently, no patient on Omnitrope developed anti-GH antibodies and 1 patient developed anti-HCP antibodies during the 12-month study.

Valtropin has been produced in Saccharomyces cerevisiae (yeast cells) by recombinant DNA technology. Valtropin was compared to Humatrope ${ }^{\circledR}$ (reference BMP) in terms of quality, safety and efficacy. The pivotal clinical trial was a double-blind, multi-centre, randomized, twoarm, 12-month parallel controlled study to compare efficacy and safety of Valtropin and Humatrope in GH-deficient children. After the end of the trial, all enrolled patients were treated for an additional 12-month period. The height velocity increase was comparable with both products, from $3.6 \pm 1.5$ and $3.4 \pm 1.1 \mathrm{~cm} /$ year at baseline to $11.3 \pm 3.0(11.2)$ and $10.5 \pm 2.8(9.6) \mathrm{cm} /$ year after 12 months of therapy with Valtropin and Humatrope, respectively (see table 2). In addition, one uncontrolled 12 -month supportive study in 30 treatment-naïve girls aged 2-9 years with short stature due to Turner syndrome was performed. The height velocity increased significantly from a baseline value of $3.75 \pm 1.76 \mathrm{~cm} /$ year to $9.73 \pm 1.55 \mathrm{~cm} /$ year. The Valtropin safety profile was consistent with that of Humatrope and with published data. There were no relevant differences in the development of anti-GH antibodies between Valtropin (2-3\%) and the reference product (2\%). Growth was not affected. The observed frequency of anti-S. cerevisiae antibodies (2\%) did not raise concern as these antibodies do not appear to have adjuvant properties, which would amplify the immune response.

\section{Areas of Uncertainties}

\section{Quality}

As any other BMP, a biosimilar BMP is in part defined by its own and specific manufacturing process (for both the active substance and the final medicinal product).
The quality attributes of the biosimilar and reference BMPs will never be identical; differences such as posttranslational modifications or process-related impurities between the two products have been frequently observed. However, it is important that any difference in the quality attributes between the two BMPs be justified in relation to its potential impact on safety and efficacy. Furthermore, current analytical methods may not be able to detect low quantities of variants or impurities, which may impact on safety and efficacy. Therefore, it is always recommended to use a final product (manufactured with the final manufacturing process) to generate the clinical data required in the guidelines. Nevertheless, as previously stated, clinical trial may not be sufficiently sensitive to detect sporadic events or events occurring after longterm exposure, and thus long-term pharmacovigilance programmes are important to confirm the absence of such events.

\section{Efficacy}

Can we extrapolate efficacy from $\mathrm{GH}$-deficient to non-GH-deficient states?

GH 'sensitivity' is viewed as higher in GH deficiency than in non-GH-deficient conditions. Therefore it could have been reasonable to require data from at least two conditions, one being GH deficiency and one non-GHdeficient conditions such as Turner syndrome. However, in the current guidelines it is considered that the demonstration of similar efficacy and safety in GH-deficient children can be extrapolated to other indications of the reference somatropin. Commercial use of approved biosimilar somatropins will allow for a global estimation of their efficacy in non-GH-deficient states. However, given the wide inter-individual variation, it is unlikely that any difference will be detectable outside the context of a randomized trial.

\section{Safety}

Are 12 Months Enough for Immunogenicity?

Given that the amplitude of the GH effect is time dependent, it would be important to know whether the therapeutic equivalence will vary over time; i.e. whether a product considered equivalent to a reference BMP after a 1-year study would still be equivalent if the study was longer or whether antibody production would change over time and influence efficacy and safety. Current guidelines propose study durations of 6-12 months for a comparative efficacy and safety assessment in children. Data on efficacy and safety after 24 months are not required in the marketing authorization file and should be made avail- 
able post-marketing. The occurrence and clinical implications of anti-rhGH and anti-HCP antibodies are closely followed as part of risk management programmes.

Are 12 Months Enough for General Safety?

The increased risk of malignancies is one of the concerns related to the increasing use of GH. For biosimilar somatropins, where only very limited information is available on IGF/IGFBP balance, a long-term prospective follow-up of patients for the development IGF-1 receptorexpressing cancers is mandatory and part of the currently proposed risk-management programmes.

\section{Substitution}

Biosimilar BMPs are authorized throughout Europe through the EMEA-centralized procedure. The subsequent possibility of substitution is the responsibility of the individual member state.

In this context it is important to remember that biosimilar BMPs are not generic medicinal products. Detected or undetected differences between the biosimilar and the reference BMP may cause differences in safety or the efficacy profile. Considering that these differences may not be observed until more experience with these biosimilar BMPs is acquired, a systematic and uncontrolled substitution, based on the prescription of the international common denomination of the active substance, does not appear reasonable at this time. In addition, biosimilar BMPs remain, in the first place, biological medicinal products with their own quality profile, related to their own manufacturing process. In principle it is not recommended to switch patients from one BMP to another. There is no reason to depart from this recommendation for a biosimilar product.
Moreover, clinical efficacy and safety of biosimilar somatropins has only been shown in one, albeit sensitive, population of patients. The extrapolation to other, less sensitive populations still needs to be proven in practice. In addition, the duration of trials was limited and possible changes in efficacy and safety in long-term use cannot be excluded. A widespread substitution would prevent detecting these potential changes.

Data on currently approved BMPs suggest that an important amount of clinical experience is necessary to obtain a thorough knowledge of the long-term safety and efficacy of these products. Therefore physicians should be involved in decisions to substitute any BMP. In this respect it is also essential to have excellent records of the treatments received in patients' clinical files, allowing physicians to trace closely the products used in case of occurrence of an adverse event.

\section{Conclusion}

The biosimilar approach allows pharmaceutical companies to submit an abridged dossier (as compared to a standard 'complete' dossier of any 'stand-alone' application) to get a marketing authorization for a biosimilar BMP, provided that a certain level of similarity is established on quality, safety and efficacy between the claimed biosimilar and reference BMPs.

Biosimilar BMPs are above all biological medicinal products, characterized by their own quality profile; the long-term consequences of these differences are not well known. Therefore, substitution of one biosimilar BMP by another is not recommended before long-term clinical efficacy and safety have been acquired in all relevant populations.

\section{References}

1 Directive 2004/27/CE du Parlement Européen et du Conseil, du 31 mars 2004; Directive 2001/83/EC, as amended by Directives 2003/63/EC and 2004/27/EC.

2 Guideline on Similar Biological Medicinal Products. CHMP/437/04 (CHMP adopted September 2005).

3 Guideline on Similar Biological Medicinal Products Containing Biotechnology-Derived Proteins as Active Substance: Quality Issues. EMEA/CHMP/BWP/49348/05 (CHMP adopted February 2006).
4 Guideline on Similar Biological Medicinal Products Containing Biotechnology-Derived Proteins as Active Substance: NonClinical and Clinical Issues. EMEA/ CHMP/42832/05 (CHMP adopted February 2006).

5 Annex Guideline on Similar Biological Medicinal Products Containing BiotechnologyDerived Proteins as Active Substance: NonClinical and Clinical Issues - Guidance on Similar Medicinal Products Containing Somatropin. EMEA/CHMP/94528/05 (CHMP adopted February 2006).
Boven K, Stryker S, Knight J, Thomas A, van Regenmortel M, Kemeny DM, et al: The increased incidence of pure red cell aplasia with an Eprex formulation in uncoated rubber stopper syringes. Kidney Int 2005;67: 2346-2353

7 Thorpe R, Swanson SJ: Assays for detecting and diagnosing antibody-mediated pure red cell aplasia (PRCA): an assessment of available procedures. Nephrol Dial Transplant 2005;20(suppl 4):iv16-iv22. 
$\checkmark 8$ Schellekens H, Ryff JC, van der Meide PH: Assays for antibodies to human interferonalpha: the need for standardization. J Interferon Cytokine Res 1997;17(suppl 1):S5-S8.

$\checkmark 9$ Bach LA, Headey SJ, Norton RS: IGF-binding proteins - the pieces are falling into place. Trends Endocrinol Metab 2005; 16: 228-234.

10 Khandwala HM, McCutcheon IE, Flyvbjerg A, Friend KE: The effects of insulin-like growth factors on tumorigenesis and neoplastic growth. Endocr Rev 2000;21:215244.

11 Jenkins PJ, Mukherjee A, Shalet SM: Does growth hormone cause cancer? Clin Endocrinol 2006;64:115-121.

-12 Johansson JO, Larson G, Andersson M, et al: Treatment of growth hormone-deficient adults with recombinant human growth hormone increases the concentration of growth hormone in the cerebrospinal fluid and affects neurotransmitters. Neuroendocrinology 1995;61:57-66.

13 Critical evaluation of the safety of recombinant human growth hormone administration: statement from the Growth Hormone Research Society. J Clin Endocrinol Metab 2001;86:1868-1870

14 Wilson TA, Rose SR, Cohen P, et al: Update of guidelines for the use of growth hormone in children: the Lawson Wilkins Pediatric Endocrinilogy Society Drug and Therapeutic Committee. J Pediatr 2003;143:415-421.

15 Cutfield WS, Wilton P, Bennmarker H, et al: Incidence of diabetes mellitus and impaired glucose tolerance in children and adolescents receiving growth-hormone treatment. Lancet 2000;355:610-613.
Blethen SL, Rundle AC: Slipped capital femoral epiphyses in children treated with growth hormone. A summary of the National Cooperative Growth Study experience. Horm Res 1996;46:113-116.

17 Wyatt D: Lessons from the national cooperative growth study. Eur J Endocrinol 2004; 151(suppl 1):S55-S59.

18 Swerdlow AJ, Higgins CD, Adlard P, Preece MA: Risk of cancer in patients treated with human pituitary growth hormone in the UK, 1959-1985: a cohort study. Lancet 2002;360: 273-277.

19 Renehan AG, O’Connell J, O’Halloran D, et al: Acromegaly and colorectal cancer: a comprehensive review of epidemiology, biological mechanisms, and clinical implications. Horm Metab Res 2003;35:712-725.

20 Orme SM, McNally RJ, Cartwright RA, Belchetz PE: Mortality and cancer incidence in acromegaly: a retrospective cohort study. United Kingdom Acromegaly Study Group. J Clin Endocrinol Metab 1998;83:27302734.

21 Renehan AG, Zwahlen M, Minder C, O’Dwyer ST, Shalet SM, Egger M: Insulinlike growth factor (IGF)-1, IGF-binding protein-3, and cancer risk: systematic review and meta-regression analysis. Lancet 2004; 363:1346-1353.
22 Lahm H, Amstad P, Wyniger J, Yilmaz A, Fischer JR, Schreyer M, Givel JC: Blockade of insulin-like growth-factor-1 receptor inhibits growth of human colorectal cancer cells: evidence of a functional IGF-II mediated autocrine loop. Int J Cancer 1994;58:452-459.

23 Burrows C, Holly JMP, Laurence NJ, et al: Insulin-like growth factor-binding protein 3 has opposing actions on malignant and nonmalignant breast epithelial cells that are each reversible and dependent upon cholesterolstabilized integrin receptor complexes. Endocrinology 2006; 147:3484-3500.

24 Chan JM, Stampfer MJ, Giovannucci E, et al: Plasma insulin-like growth factor-1 and prostate cancer risk: a prospective study. Science 1998;279:563-566.

25 Juul A, Bernasconi S, Jarel JC, Clayton PE, Kiess W, DeMuinck-Keizer Schrama S: Growth hormone treatment and risk of solid tumours. A statement for the Drugs and Therapeutics Committee of the European Society for Paediatric Endocrinology. Horm Res 2003;60:103-104.

26 Omnitrope, European Public Assessment Report, 12 April 2006, http://www.emea.europa.eu/humandocs/Humans/EPAR/omnitrope/omnitrope.htm.

27 Valtropin, European Public Assessment Report, 24 April 2006, http://www.emea.europa.eu/humandocs/Humans/EPAR/valtropin/valtropin.htm.

28 Omnitrope (somatropin) questions and answers. US Food and Drug Administration, Center for Drug Evaluation and Research. http://www.fda.gov/cder/whatsnew.htm. 